\title{
FU Orionis eruptions and early stellar evolution
}

\author{
Bo Reipurth \\ European Southern Observatory \\ Casilla 19001 \\ Santiago 19 \\ Chile
}

\begin{abstract}
The FU Orionis phenomenon has attracted increasing attention in recent years, and is now accepted as a crucial element in the early evolution of low mass stars. The general characteristics of FUors are outlined and individual members of the class are discussed. The discovery of a new FUor, BBW 76, is presented, together with a discussion of photometric and spectroscopic observations of the star. The evidence for circumstellar disks around T Tauri stars is briefly outlined, and the FUor phenomenon is discussed in the context of a disk accretion model. A large increase in the accretion rate through a circumstellar disk makes the disk self-luminous with a luminosity two or more orders larger than that of the star. Massive cool winds rise from FUors, and it is conceivable that they are related to the initiation of Herbig-Haro flows. The FUor phenomenon appears to be repetitive, and newborn low-mass stars may be cycling between the FUor state and the T Tauri state.
\end{abstract}

\section{The FUor phenomenon}

The story of FU Orionis eruptions began in 1936, when a faint red variable star, FU Orionis, located in the dark cloud B35 in the $\lambda$ Ori region, brightened by 6 magnitudes in a period possibly as short as four months. Early photometry has been discussed by Wachmann $(1939,1954)$ and a detailed photometric and spectroscopic analysis is presented by Herbig $(1966,1977)$.

There are now five such objects known, dubbed FUors by Ambartsumian (1971), which all have in common that they were observed to brighten considerably, although on rather different time scales. In their bright states, these stars share a number of spectroscopic properties, notably F-G type supergiant spectra with abnormally strong Balmer absorption lines, strong Li I 6707 lines, pronounced P Cygni profiles at $\mathrm{H} \alpha$ and several other lines, and broad lines suggesting rapid rotation. They are all located in regions of recent star formation, and are associated with reflection nebulae. After 
reaching maximum brightness they presumably gradually decay, although only one object (V1057 Cyg) has faded substantially since its eruption (Fig. 1); since we are not seeing numerous objects in high states it follows that all must eventually decay.

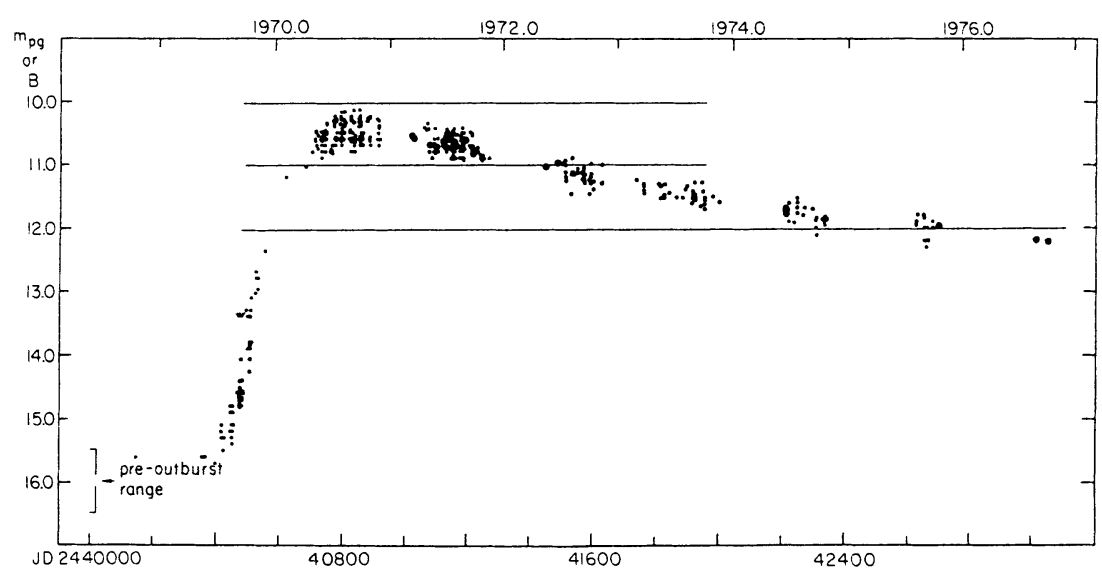

Fig. 1. The B-light curve of V1057 Cyg showing the eruption in $1969 / 70$ and the subsequent decay. From Herbig (1977).

The progenitors of FUors are faint, red, slightly variable stars, all presumably $\mathrm{T}$ Tauri stars, although for only V1057 Cyg is a pre-outburst spectrum available (Herbig 1958); this spectrum shows the emission lines characteristic of T Tauri stars.

Based on event statistics and the estimated number of observable T Tau stars, Herbig (1977) demonstrated that FUor eruptions almost certainly occur repetitively in all T Tau stars in the course of their evolution. (see Section 8).

Perhaps the most outstanding spectroscopic characteristic of FUors is their distinct $\mathrm{P}$ Cygni profiles, notably at $\mathrm{H} \alpha$ and at the $\mathrm{Na}$ I 5890/5896 lines (Bastian and Mundt 1985). Fig. 2 shows high-resolution spectra of these lines in FU Ori. Deep blueshifted absorption troughs testify to the presence of massive stellar winds in these objects (for further details see Sec. 6). Another notable feature of FUors is their gradual change of spectral type with wavelength: in the red spectral region they have a later spectral type than in the blue, and in the near-infrared they show the CO bands typical of K-M stars (Cohen 1975, Mould et al. 1978).

On the basis of such spectroscopic characteristics, several stars have been suggested as FUors, without evidence for an eruption, in particular Z CMa and L1551 IRS 5. These two objects are discussed, together with the five known classical FUors, in the following.

\section{- FU Orionis}

The photometric behaviour of FU Ori from 1936 to 1977 has been compiled by Herbig (1977), and from 1978 to 1985 by Kolotilov and Petrov (1985). During that 
time the star has remained in its elevated state, with only a slight overall fading. Two years after the eruption the star suddenly dimmed by a magnitude, and subsequently started a slow oscillation which ceased after 20 years. Overall the star has faded by about a magnitude in the blue since the eruption.
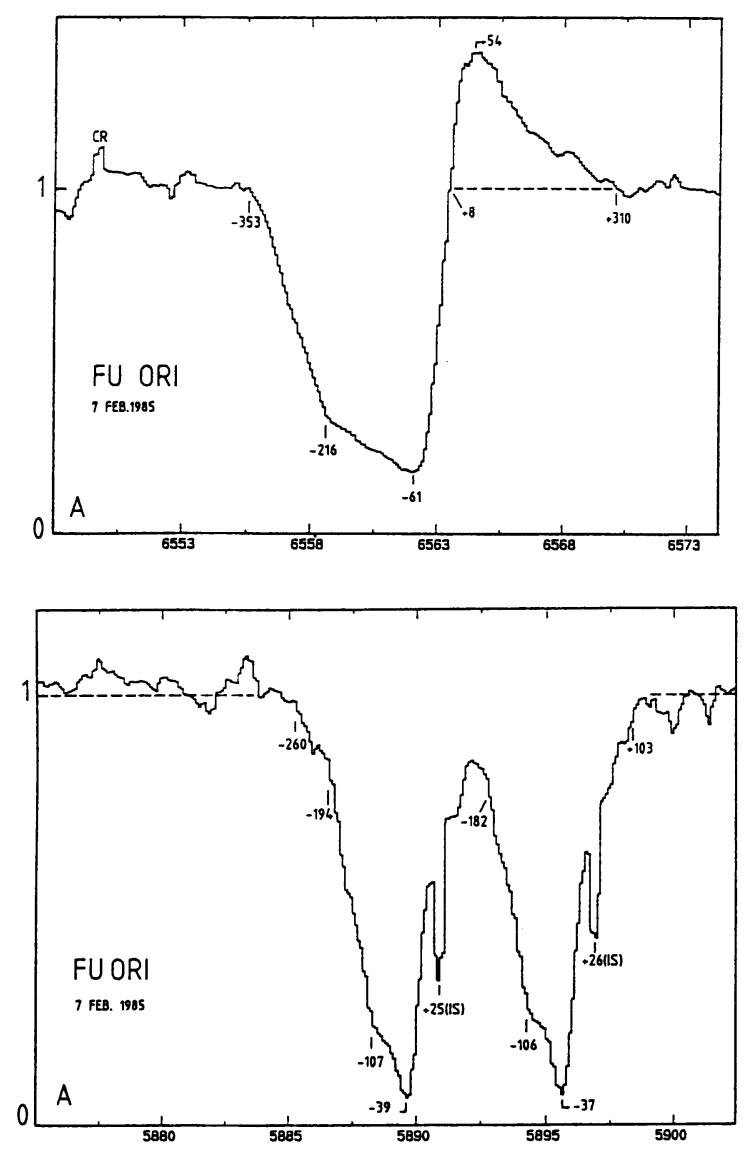

Fig. 2. High-resolution line profiles of the $\mathrm{H} \alpha$ and the $\mathrm{Na} I$ lines in FU Orionis, showing evidence for massive winds. From Reipurth (1990).

The peculiar composite spectrum of FU Ori has been described in detail by Herbig (1966), who also gives references to the early work on this object. At low resolution the star appears as an F-G-type supergiant, but with unusually prominent Balmer lines. At higher resolution one sees two components, the above mentioned supergiant spectrum as well as a set of blueshifted components particularly strong at the Balmer lines and at low-level lines in both neutral and ionized metals. The best compromise classification of the supergiant spectrum is F2:p I-II (Herbig 1966). 
The far-red spectrum has been described by Shanin (1979), and ultraviolet spectra by Ewald et al. (1986) and Kenyon et al. (1989).

FU Ori is a bright near-infrared source (e.g. Cohen 1973) and is also detectable at far-infrared wavelengths with a dust-temperature of $15 \mathrm{~K}$ (Smith et al. 1982). The luminosity in the near-infrared interval 1.2 to $5 \mu \mathrm{m}$ is about $80 \mathrm{~L}_{\odot}$ and it is about $20 \mathrm{~L}_{\odot}$ in the IRAS interval from 12 to $100 \mu \mathrm{m}$, assuming a distance of $460 \mathrm{pc}$.

The bright-rimmed cloud B35 has a cometary appearance. It harbors several cloud cores detected in CO (Lada and Black, 1976); FU Ori is located towards the tail of the cloud, outside the main cores.

\section{- V1057 Cyg}

The extensive molecular clouds in the NGC 7000 (North America Nebula) region contain numerous $\mathrm{H} \alpha$ emission stars, catalogued by Herbig (1958). In 1969-1970, one of these, $\mathrm{LkH} \alpha 190$, suddenly erupted and brightened by about 5 magnitudes in a time-span less than 400 days (Welin 1971). A pre-outburst spectrum taken by Herbig (1958) showed an emission line spectrum characteristic of T Tauri stars. Posteruption spectra show a great similarity to FU Ori, with supergiant characteristics and a spectral type varying with wavelength; Herbig (1977) classified the star in the blue (from the metallic-line spectrum) as changing from A3-5 II to early F, II or III, from 1971 to 1973 , and in the red from F5II to G0Ib during the same period. Other early post-eruption spectra are discussed by Haro (1971), Mendoza (1971), Schwartz and Snow (1972), Gahm and Welin (1972), Grasdalen (1973) and Chalonge et al. (1983). The $\mathrm{H} \alpha$ line shifted considerably in velocity as measured on mediumresolution spectra in the years after the eruption, lessening from $-560 \mathrm{~km} / \mathrm{sec}$ in early 1971 to about $-220 \mathrm{~km} / \mathrm{sec}$ in 1975 (Herbig 1977), with subsequent variations between -200 and $-300 \mathrm{~km} / \mathrm{sec}$, as measured until 1982 by Kolotilov (1984). A P Cygni profile was observed in 1974 at the He I 10830 line, in which the emission disappeared and the absorption weakened and shifted to lower outflow velocities in the following two years (Shanin 1979). Obviously the eruption was accompanied by a brief period of enhanced mass loss of exceptionally high velocity. High resolution spectra of the $\mathrm{H} \alpha$ and Sodium D lines continue to show evidence for high velocity mass loss (Bastian and Mundt 1985, Croswell et al. 1987), very similar to what is observed in FU Ori (see Fig. 2 and Section 6).

The optical light curve of V1057 Cyg is well observed. Fig. 1 shows the monotonic decline after the eruption until 1977 (Herbig 1977); further photometry up to 1985 has been obtained by Kopatskaya (1984) and Ibragimov and Shevchenko (1987). The star is now almost halfway back to its average pre-outburst level, but the rate of decrease in brightness appears to be slowing down. Ibragimov and Shevchenko (1987) suggest that there could be a cyclic variation in their photometry with a period around 12 days. The decline in brightness of V1057 Cyg has been accompanied by a corresponding fading of the extensive reflection nebulosity around the star (Duncan et al. 1981)

Infrared observations of V1057 Cyg obtained in the seventeen years following 
outburst have been analyzed by Simon and Joyce (1988), who also give reference to earlier infrared work. The infrared fluxes have decreased at all wavelengths observed between 1.2 and $20 \mu \mathrm{m}$, but much more rapidly at the shorter wavelengths. At 10 and $20 \mu \mathrm{m}$ an initial very rapid fading subsequently leveled off. To explain the rate of decrease as a function of wavelength is an essential test for any model of the FUor phenomenon.

In 1973 a $1720 \mathrm{MHz}$ OH maser was observed at V1057 Cyg, which gradually faded beyond detection towards the end of 1974 (Lo and Bechis 1973, 1974; Andersson et al. 1979). A second $\mathrm{OH}$ maser outburst was reported by Winnberg et al. (1981) to occur in 1979 , also with a subsequent fading.

V1057 Cyg was detected at 450,800 and $1100 \mu \mathrm{m}$ by Weintraub et al. (1989) providing direct evidence for the presence of circumstellar material; they suggest a lower limit to the mass of this material of $10^{-1} M_{\odot}$.

\section{- V1515 Cyg}

The third FUor, V1515 Cyg, was discovered by Herbig (1977) in the Lynds 897 cloud, a complex region with various signs of recent star formation (Herbig 1960). In contrast to the rapid rise displayed by FU Ori and V1057 Cyg, V1515 Cyg took several decades to rise: from 1944 to 1952 it brightened by about 3 mag from $\mathrm{m}_{B}$ around $17^{m} \cdot 5$, followed by a slower rise until the mid-seventies when it reached $\mathrm{V} \simeq 12^{m}$ (Herbig 1977, Gottlieb and Liller 1978). Since then it appears to have started to fade (Kolotilov and Petrov 1983). It displays the various characteristics of FUors, such as an early G-type high-luminosity spectrum, a bow-shaped reflection nebula (see Fig. 4), deep blueshifted absorptions at $\mathrm{H} \alpha$ and the Sodium lines (Bastian and Mundt 1985), and a strong infrared excess (Cohen 1980).

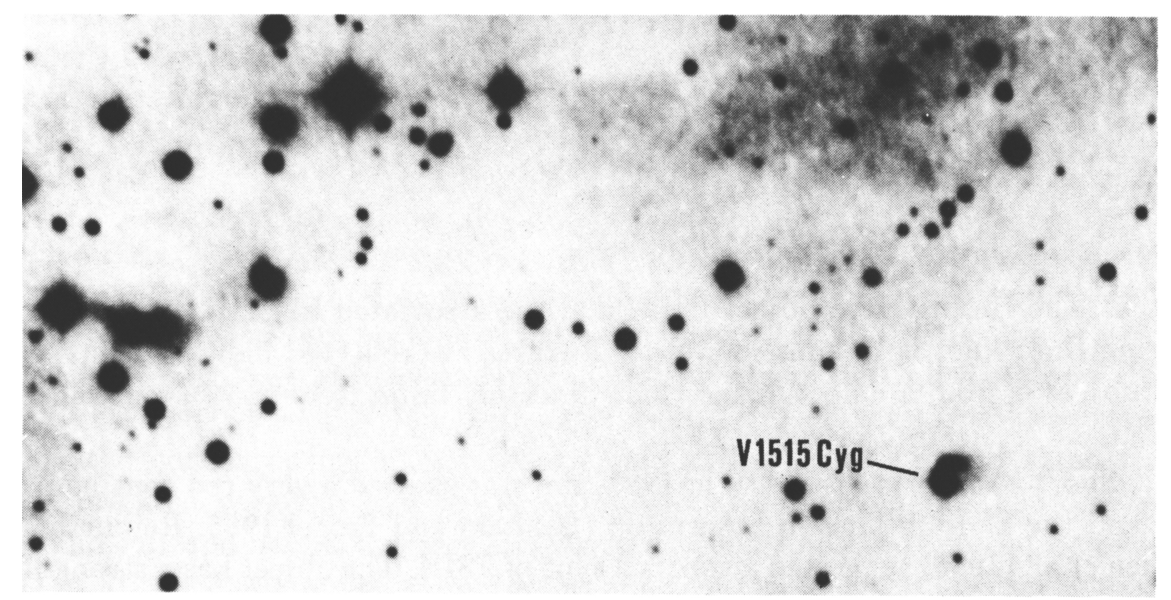

Fig. 3. The FUor V1515 Cyg and its environment, as seen on the red Palomar atlas. The characteristic FUor reflection nebula is evident. Another young star, Parsamian 22 , is seen further east in an extended reflection nebula. North is up and east is left. 


\section{- V1735 Cyg (=Elias 1-12)}

This object was found as a strong infrared source in an infrared survey of the IC 5146 dark cloud complex (Elias 1978). It is a bright near-infrared source rising steeply towards the mid-infrared. It is also a bright IRAS source, with an IRAS luminosity of $75 L_{\odot}$ at the assumed distance of $900 \mathrm{pc}$. On the red Palomar atlas plate from 1952 , no object brighter than $20^{m}$ was visible, but a similar plate from 1965 showed a star of about $15^{m}$ with a nearby faint reflection nebula. An optical low-resolution spectrum shows a red continuum with $\mathrm{H} \alpha$ in absorption, displaced $4 \AA$ bluewards, suggesting a $\mathrm{P}$ Cygni profile. The near-infrared spectrum shows strong $\mathrm{H}_{2} \mathrm{O}$ and $\mathrm{CO}$ absorption features, as in other FUors. CO line profiles of the surrounding cloud show broad wings, suggesting outflow from the star (Levreault 1983).

\section{- V346 Nor (= HH 57 IRS)}

The most recent FUor to be discovered is V346 Nor, which appeared in a molecular cloud in the southern constellation Norma, next to the Herbig-Haro object HH57 (Graham 1983, Graham and Frogel 1985). Fig. 4 shows a red broadband CCD image of the region, which demonstrates the close association between $\mathrm{HH}$ object and FUor: a reflection nebula stretches out from the star to reach the $\mathrm{HH}$ object. The proper motion vector of the HH object points away from the FUor (Schwartz et al. 1984).

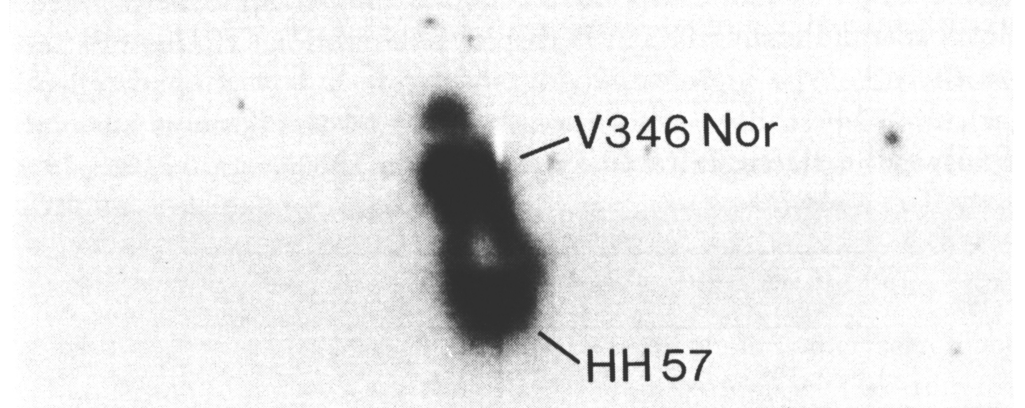

Fig. 4. The Herbig-Haro object HH57 and the associated FUor V346 Nor. Only the Herbig-Haro knot is in emission, other features are reflected light. The spike in the $\mathrm{HH}$ object is from a defect in the CCD detector. From Reipurth (unpublished).

Shortly after its discovery in 1983, the star showed a very red continuum, with $\mathrm{H} \alpha$ and the Sodium lines in absorption (Graham and Frogel 1985, Reipurth 1985a). Cohen et al. (1986) suggested a spectral type of F8III. The object has a strong infrared excess. In the far-infrared there is an elongated structure perpendicular to the line between $\mathrm{HH}$ object and star (Cohen et al. 1985). A molecular outflow from the FUor has been detected along the axis to the HH object (Reipurth et al. 1990). 
Little is known about the rise time. Examination of Schmidt plates suggest a slower rise than FU Ori and V1057 Cyg, more reminiscent of V1515 Cyg (Reipurth 1985a).

The luminosity is poorly determined, mainly because the distance is very uncertain. Various distance estimates are 300 pc (Reipurth 1985a), 700 pc (Graham and Frogel 1985) and $940 \mathrm{pc}$ (Cohen et al. 1986). The last estimate is derived assuming the FUor has the same luminosity as FU Ori at peak brightness.

\section{- Z Canis Majoris}

This object has long been considered a Herbig Ae/Be star, but it possesses a number of characteristics which suggest it may be a FUor (Hartmann et al. 1989): it exhibits strong blueshifted Balmer and Sodium absorption troughs (Finkenzeller and Jankovics 1984, Finkenzeller and Mundt 1984), a unique spectral type cannot be assigned (Herbig 1960), CO absorption at $2.3 \mu \mathrm{m}$ (Hartmann et al. 1989), and a characteristic reflection nebula (Herbig 1960, Goodrich 1987). It also shows doublepeaked absorption line profiles (Hartmann et al. 1989), as seen in other FUors and predicted by the accretion disk model (see Sect. 5).

No outburst has been observed, neither is $\mathrm{Z}$ CMa known to fade gradually, instead it shows irregular variability with amplitudes up to $\Delta V \sim 2 \mathrm{mag}$ (e.g. Covino et al. 1984).

Near-infrared speckle observations have shown $\mathrm{Z} \mathrm{CMa}$ to be highly elongated with a size of about $0.1^{\prime \prime}$ at $2.2 \mu \mathrm{m}$, either due to scattering in a circumstellar disk (Leinert and Haas 1987) or because of a close companion (Koresko et al. 1989). It has also been resolved at radio continuum wavelengths (Cohen and Bieging 1986). The close visual companion reported by Finkenzeller and Mundt (1984) appears to be spurious.

It is likely that $\mathrm{Z} \mathrm{CMa}$ is a FUor. With a luminosity of $3500 L_{\odot}$ it is more luminous than any of the other known FUors.

\section{- L1551 IRS 5}

This object is an infrared source embedded in the L1551 cloud (Strom et al. 1976) with a luminosity of $36 L_{\odot}$ (Cohen and Schwartz 1987). It is presumably very young, drives a major bipolar molecular outflow (Snell et al. 1980), and a series of Herbig-Haro objects are moving supersonically away from it (Cudworth and Herbig 1979, Neckel and Staude 1987). High resolution $\mathrm{C}^{18} \mathrm{O}$ observations have revealed an elongated concentration of gas, with a long axis of $1400 \mathrm{AU}$ and a mass of $0.1 M_{\odot}$, oriented perpendicular to the outflow-axis (Sargent et al. 1988). A small optical and infrared nebula outlines a cavity reflecting the light from the source (e.g. Moneti et al. 1988, Campbell et al. 1988). The optical spectrum of the source has been observed by Mundt et al. (1985) and Stocke et al. (1988) via this reflection nebula. They find a $\mathrm{P}$ Cygni profile at $\mathrm{H} \alpha$, with blueshifted absorption extending to more than $500 \mathrm{~km} / \mathrm{sec}$, and only weak $\mathrm{H} \alpha$ emission. This is unlike the $\mathrm{H} \alpha$ profiles 
of T Tauri stars, but similar to what is observed in FUors. There is some tentative evidence for a modest change in spectral type as one goes from blue to red, from $G$ to K. Carr et al. (1987) observed the $2 \mu \mathrm{m}$ spectrum of L1551 IRS 5 and detected the $\mathrm{CO}$ bands in absorption at a strength suggesting a spectral type of K2III. They also find that $\mathrm{H}_{2} \mathrm{O}$ absorption is possibly present. The sum of the observational evidence thus points to L1551 IRS 5 being in a post-eruption FUor state.

\section{BBW76 - a new southern FUor}

The photometric behaviour of the FUors discussed in the previous section show large differences. In order to better discern common properties from individual characteristics, it would be very useful to enlarge the small sample of known FUors. This can be done in two ways: either by searching for major stellar eruptions in molecular cloud regions in new or older plate material, or by closer examination of individual nebulous stars in star forming clouds. This latter approach presupposes that FUors have some sufficiently well defined characteristics, which allow them to be identified without evidence for an outburst. As discussed in the previous section, this appears in certain cases to be possible.

Both types of studies have been carried out at the European Southern Observatory at La Silla over the last 10 years, and one object has been identified as an apparently bona fide FUor (Reipurth 1985b, 1990). The star, BBW 76, is a $12^{\text {th }}$ magnitude object associated with a small, anonymous cloud in the direction of the constellation Puppis at a position $\alpha_{1950}: 7^{h} 48^{m} 40^{s} .4, \delta_{1950}:-32^{\circ} 58^{\prime} 43^{\prime \prime}$. The star and cloud are apparently associated, because the star displays a distinct, curved reflection nebula. Also, the velocity of the cloud as measured in the ${ }^{12} \mathrm{CO}$ transition equals the velocity of the star, as well as of the interstellar absorption lines seen in high resolution $\mathrm{Na}$ I spectra.

Low resolution spectra of the star reveal no emission lines, but a composite of several types of features, mixing a host of metallic lines, strong Balmer lines and molecular bands. Based on a blue spectrum, and excluding the Balmer lines, the best spectral type is G0-2 I. Fig. 5 shows a low-resolution spectrum of BBW 76 compared to one of FU Ori itself. The similarity of the two stars is striking. Further to the red the two stars are equally similar, and both show strong Li I 6707 absorption. In the near-infrared region, BBW 76 shows weak $2.3 \mu \mathrm{m}$ CO absorption, suggestive of a much later spectral type.

At much higher resolution, the similarities between BBW 76 and FU Ori continue. Fig. 6 shows high-resolution echelle spectra of $\mathrm{H} \alpha$ and the $\mathrm{Na}$ I 5890/5896 lines in BBW 76. Comparison with similar spectra for FU Ori shown in Fig. 2 immediately suggests that similar processes operate in both stars. $\mathrm{H} \alpha$ displays a $\mathrm{P}$ Cygni profile with weak emission and a deep absorption trough several hundred $\mathrm{km} / \mathrm{sec}$ wide. The sodium lines also show massive blueshifted absorption features, which are structured, 
suggesting spatial/temporal variability. The winds responsible for these features are further discussed in section 6 .

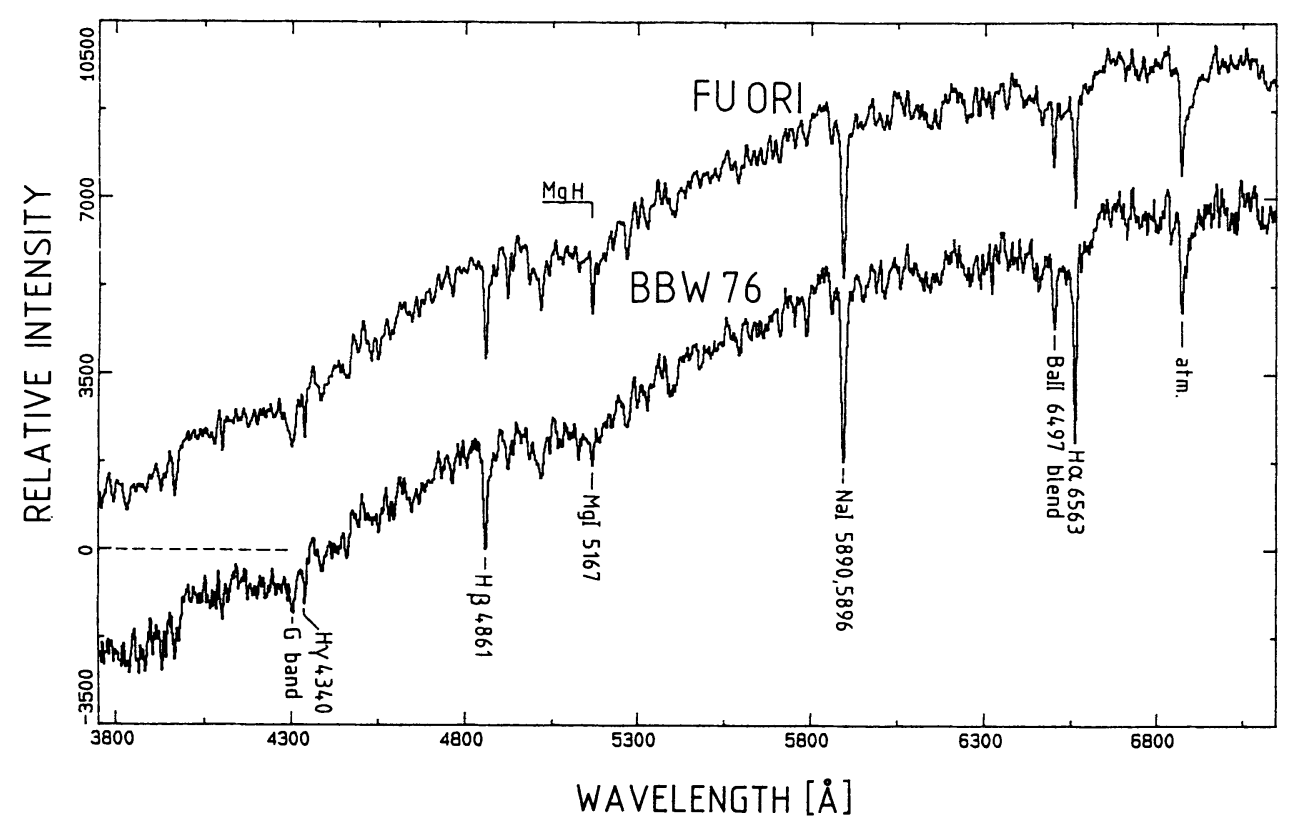

Fig. 5. Low resolution spectra of BBW 76 and FU Orionis, showing the remarkable similarity between the two objects. From Reipurth (1990).

If BBW 76 is a FUor decaying from an earlier eruption, it should exhibit a gradual fading at all wavelengths. Photometric observations, at optical as well as infrared wavelengths, demonstrate that this is indeed the case. Fig. 7 shows the photometric behaviour in the V-band of BBW 76 between 1983 and 1988. To within the photometric accuracy the star is fading steadily at a rate of about $0^{m} .025 \mathrm{yr}^{-1}$. This is a rate almost twice as fast as FU Ori, but five times slower than V1057 Cyg.

The evidence presented so far strongly suggests that BBW 76 is a FUor. The piece of information missing is the date of its initial eruption. A still ongoing survey of old plate archives has revealed no major variability going back as early as 1927 .

In summary, BBW 76 is a young star, with strong lithium and associated with a molecular cloud, which spectroscopically shares all the features of FU Ori itself, and which is gradually fading at optical and infrared wavelengths. If a major brighteningof the star has occurred, it must have taken place more than 60 years ago. 

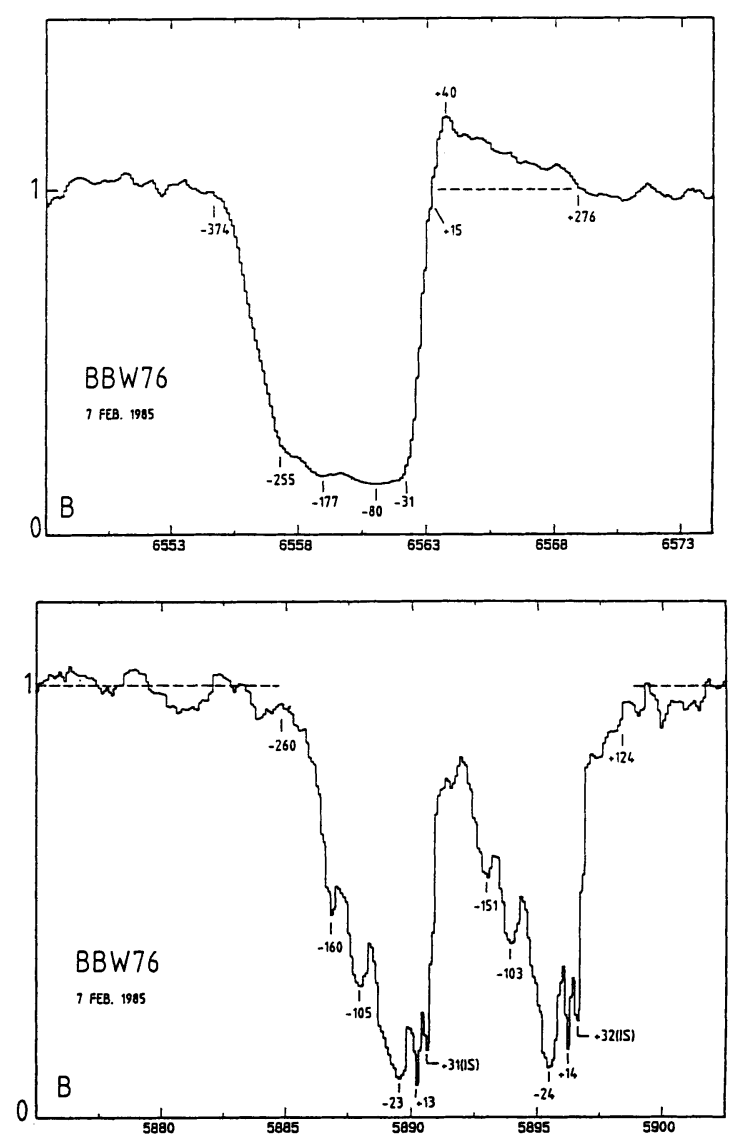

Fig. 6. High resolution line profiles of the $\mathrm{H} \alpha$ and $\mathrm{Na}$ I lines in the new FUor BBW 76, showing the same signatures of mass loss as in FU Orionis. From Reipurth (1990).

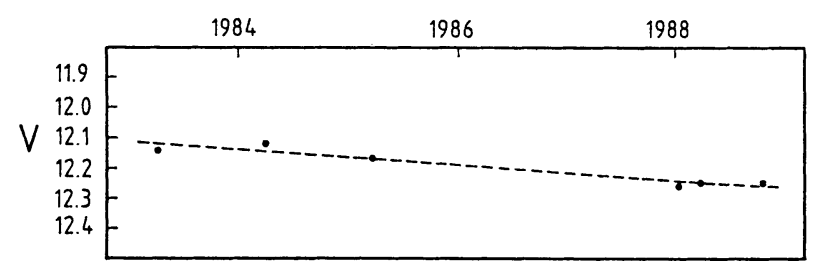

Fig. 7. The V-light curve of the new FUor BBW 76 from 1983 to 1989 . The object is clearly fading; a similar behaviour is found at near-infrared wavelengths. From Reipurth (1990). 


\section{EXors}

Herbig (1977) pointed out that if FU Ori eruptions "occur in T Tauri stars but are absent on the main sequence, then in a given object they probably weaken in intensity, duration, or frequency as the star evolves toward the main sequence, instead of ceasing abruptly. If so, minor activity reminiscent of the FU Ori phenomenon might be present in older T Tau stars or post-T Tau objects." It is also possible that the mechanism which drives FUors may operate at lower levels, also in very young stars.

A number of objects are known that fit this description; they are known as EXors, and a list of nine members of the class is given by Herbig (1989).

The EXors, named after the prototype EX Lupi, show outbursts with an amplitude of up to 5 magnitudes, which last for months, in some cases more than a year. They do, however, appear to retain their emission line characteristics also during outbursts, although spectroscopic data are limited. A light curve of the 1955-56 outburst in EX Lupi is shown in Fig. 8.

The best-studied EXor to date is probably DR Tau, a T Tauri star with a rich emission line spectrum and rapid high-amplitude photometric variability superposed on a long term rise in mean brightness (Bertout et al. 1977, Chavarria 1979, Götz 1980, Kolotilov 1987).

The two most recently found EXors are V1118 Ori (= Chanal's object) and V1143 Ori (= Sugano's object). They have had several outbursts in the 1980's, which are discussed by Parsamian and Gasparian (1987), Gasparian and Ohanian (1989), Mirzoyan et al. (1988); see also Parsamian (this volume). Photometric and spectroscopic monitoring of these stars is important for attempts to understand their relationship with the FUor phenomenon.

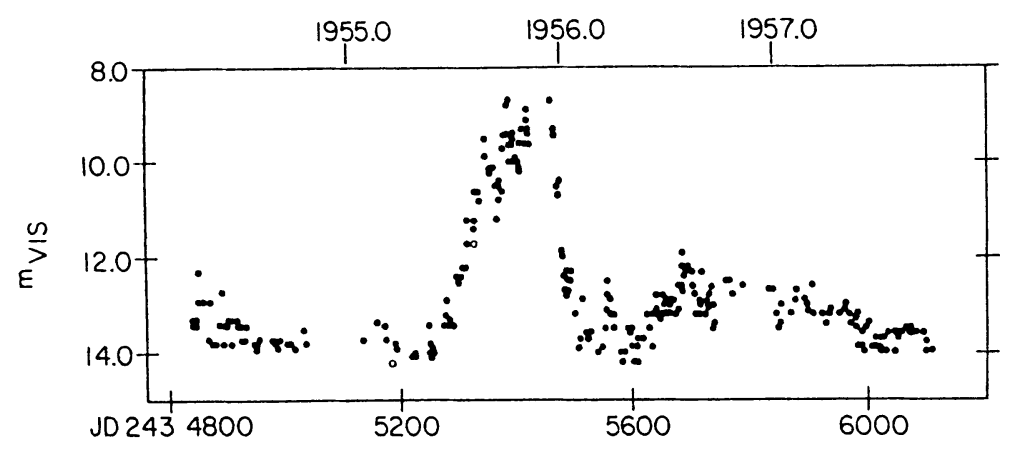

Fig. 8. The 1955/56 eruption of the EXor EX Lup. The data points are visual estimates compiled by Bateson and Jones. From Herbig (1977). 


\section{Disks around $\mathbf{T}$ Tauri stars}

It is now generally accepted that circumstellar disks not only are present around the younger of low-mass pre-main sequence stars, but play an essential part in defining a variety of their observable properties. This section briefly summarizes properties of disks around the T Tauri stars, in their role as precursors to FUors (see also the reviews of Appenzeller and of Lada in this volume).

$\mathrm{T}$ Tauri disks are divided into passive and active disks. The passive disks merely reprocess light emitted from the central star, re-emitting it in the infrared. For certain geometries and inclinations, up to $50 \%$ more light than emitted by the star can actually reach the observer via scattering. On the other hand, active disks have an additional component to their luminosity from viscous dissipation of energy in the disk, and their infrared excesses can therefore occasionally be very large. As discussed by Lynden-Bell and Pringle (1974), energy dissipation must lead to inflow of material through the disc; active disks are therefore accretion disks. Stars with active disks are likely to have a hot boundary layer between star and disk, where energy is released as disk material comes to rest on the (slower rotating) star.

The spectral shape of infrared energy distributions is almost identical for $\mathrm{T}$ Tauri stars with passive and active disks. For a detailed modelling, optical and ultraviolet data must therefore be incorporated, even though the effect of extinction then becomes much more pronounced. Bertout et al. (1988) and Basri and Bertout (1989) have rather successfully modelled the energy distributions of a number of classical T Tauri stars from the ultraviolet to the infrared in terms of a central star surrounded by an active disk. Such accretion disk models are successful in matching observations, because the infrared radiation can be produced by dust near the star without causing an excessive extinction, and because accretion is a convenient source of energy to explain a possible large infrared luminosity. The accretion rates they derive range from $10^{-9} M_{\odot} y r^{-1}$ to $10^{-7} M_{\odot} y r^{-1}$.

Certainly not all young low mass stars show evidence that they are surrounded by actively accreting disks. Estimates differ, but it appears that less than $50 \%$ of the classical T Tauri stars show signatures of an accretion disk.

Age and mass determinations of $\mathrm{T}$ Tauri stars are generally made using pre-main sequence evolutionary tracks. This is in itself a very uncertain procedure (Mazzitelli 1989), but with the growing realization that disks can contribute sometimes significantly to the observed luminosities, such determinations become questionable (Kenyon and Hartmann 1990).

T Tauri stars are known to possess powerful winds. Several recent studies have suggested correlations between wind diagnostics and evidence for disks. Strom et al. (1988) found that most of young stellar objects with signatures of mass loss are characterized by large infrared luminosity excesses. Cohen et al. (1990) found that stars with strong [OI] emission have bolometric luminosities much larger than their expected stellar luminosities. Cabrit et al. (1990) found [OI] and $\mathrm{H} \alpha$ emission correlated with infrared excess, but no correlation to photospheric luminosity, suggesting 
that it is the disk, and not the star, which primarily determines the stellar wind strength. It thus appears that mass loss in young low-mass stars is powered by the energy released as material in circumstellar disks accretes onto the stars.

\section{Disk accretion in FUors}

A number of authors have suggested over the years that the large flare-ups of FUors could represent events in which material accretes onto the central stars (Paczynski 1976, Herbig 1977, Larson 1983, Lin and Papaloizou 1985). Recently, Hartmann and Kenyon (hereafter HK) have developed the details of an accretion disk model for FUors, in which a hot, optically-thick accretion disk dominates the system light at maximum (Hartmann and Kenyon 1985, 1987a, b, Kenyon et al. 1988, Kenyon and Hartmann 1989).

The relations between mass accretion, angular momentum transport and energy dissipation in a viscous disk have been discussed by Shakura and Sunyaev (1973) and Lynden-Bell and Pringle (1974). The effect of viscosity is to transport angular momentum from the inner to the outer part of the disk and thus to control the mass-flow through the disk. While material slowly drifts inwards in the disk, half of the potential energy decrease is used to heat the disk locally. Ultimately the speeding material accretes onto the star, releasing the equivalent of the other half of the potential energy difference in a hot boundary layer. The local temperature and luminosity in the disk is a sensitive function of the mass accretion rate and distance to the star.

Based on these concepts, HK developed a time-independent model of an optically thick disk, consisting of a set of concentric annuli, each of them radiating as a star with a certain effective temperature, or for the outer cool regions, as a black body.

The interpretation of FUors in terms of such a hot, optically thick accretion disk has had some notable successes. It naturally accounts for the otherwise curious change of spectral type with wavelength, wherein a FUor has an F-G type spectrum in the blue range, but a $\mathrm{K}-\mathrm{M}$ type spectrum in the near-infrared. It further explains the supergiant spectra of FUors, because disks are low-gravity environments. It also makes two predictions, which can be tested by observations.

The first prediction is that absorption-line profiles should be double-peaked if the object observed is a rotating flattened disk. High resolution optical spectra do not show this effect unambiguously in individual lines. But cross-correlation between FUor spectra and spectra of template stars, of similar spectral type in the relevant wavelength range, indeed shows such double peaked profiles in FU Ori, V1057 Cyg and $\mathrm{Z} \mathrm{CMa} \mathrm{(HK,} \mathrm{see} \mathrm{previously} \mathrm{listed} \mathrm{references).} \mathrm{The} \mathrm{linewidths} \mathrm{are} \mathrm{large}(\sim 100$ $\mathrm{km} \mathrm{s}^{-1}$ ) and the separation of the double peaks can be well matched by disk models.

The second prediction is that lines from optical spectra, emitted in hot, inner, 


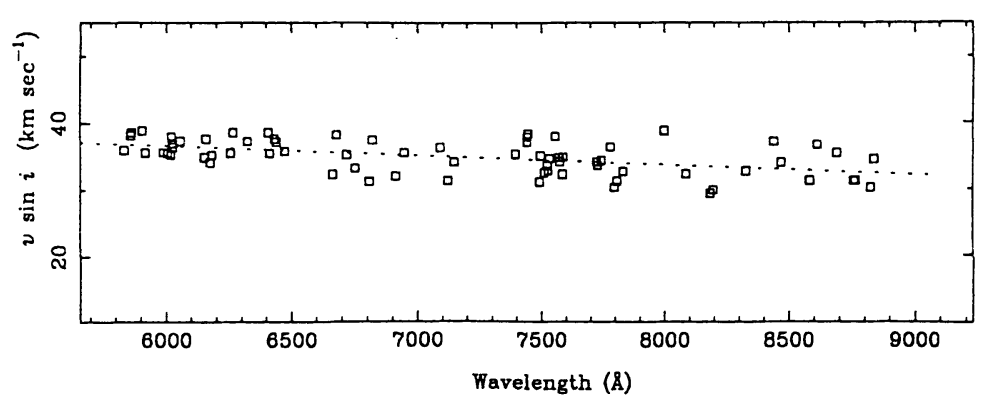

Fig. 9. The rotational velocity $v$ sin $i$ determined for a large number of lines in the red spectral region $(6000-9000 \AA)$ of V1057 Cyg. There is a clear trend towards lower rotational velocities at longer wavelengths. From Welty et al. (1990).

faster rotating regions, should show larger rotational broadening than lines from infrared spectra, emitted in cooler, more distant and slower rotating regions. Using infrared Fourier transform spectra, HK (Kenyon and Hartmann 1989 and previously listed references) have shown that there is indeed such an effect, and that the observed ratio of optical to infrared rotational broadening can be well reproduced by the disk model. In a more recent study, Welty et al. (1990) obtained high resolution, high signal-to-noise spectra of V1057 Cyg covering the spectral range from $5820 \AA$ to 9370 $\AA$. Fig. 9 shows $v \sin i$ measured for a number of lines in this wavelength region. The trend towards lower rotational velocity as one goes to the red is evident, and further supports the disk accretion hypothesis. In the same study, Welty et al. (1990) also found a correlation between line width and line transition lower excitation potential, such that higher excitation potential lines are broader, as one would expect in a Keplerian disk with a temperature gradient.

In order to achieve a match between models and observations, very large mass accretion rates, of the order of $10^{-4} M_{\odot} \mathrm{yr}^{-1}$, are needed. Assuming an average duration of a FUor eruption of 100 years, thus implies that of the order of $10^{-2} M_{\odot}$ is accreted in an eruption. Although these numbers obviously are nothing more than simple estimates, they do suggest that if FUor eruptions occur regularly through the pre-main sequence phase, the accreted material may add significantly to the initial stellar mass, and thus influence the evolution of the star.

Despite the ease with which a number of observations can be interpreted in this framework, the accretion disk model is still in its infancy, and it is likely to need considerable elaboration before it can explain a number of more subtle observational facts. Herbig (1989) has drawn attention to some of the shortcomings of the model as it stands at the moment, and suggests that it is still too early to discard other alternatives.

A point of considerable interest is the question of stability of a hot actively accreting circumstellar disk. Clarke et al. (1989) note that the mid-plane temperature 
in the inner disk is at least $40000 \mathrm{~K}$, and therefore must contain partially ionized regions. But such regions are prone to local thermal instability, so that a front separating ionized and neutral regions should sweep radially back and forth in the disk, modulating the otherwise steady accretion on timescales of months to years. This is contrary to the observed steady decline of FUors. Clarke et al. (1989) proceed to show that the disk may actually be stabilized against this thermal ionization instability by the effect of advective heat transport.

A problem not yet understood is what initially triggers a FUor outburst. It is difficult to envisage how a disk around a T Tauri star, with an accretion rate of less than $10^{-7} M_{\odot} y r^{-1}$, can switch into a structure with an accretion rate 3 orders of magnitude larger within a few years. An external triggering mechanism may be needed, such as the close passage of a companion in a highly eccentric orbit (Clarke et al. 1989).

\section{The winds of FUors}

High-resolution spectra of $\mathrm{H} \alpha$ and the Sodium D lines demonstrate that FUors possess massive, high velocity winds (Bastian and Mundt 1985, Croswell et al. 1987, Reipurth 1990). Profiles of these lines are shown for FU Ori and the new FUor BBW76 in figs. 2 and 6 , respectively. In $\mathrm{H} \alpha$ the emission component extends to only low positive velocities, in contrast to the absorption trough which extends to blueshifted velocities of several hundred $\mathrm{km} / \mathrm{sec}$. In the Sodium lines, the absorption also extends to very high negative velocities, whereas only weak redshifted absorption of low velocity can sometimes be discerned. Apparently the redshifted material is somehow occulted. This is readily understood if an optically thick disk surrounds the star.

Pudritz and Norman $(1983,1986)$ suggested that winds from young stellar objects originate in their circumstellar disks. A disk is an attractive wind-source, because its surface-gravity is much lower than that of the central star, and during a FUor eruption it is also many times more luminous than the star. The PudritzNorman model assumes that poloidal magnetic fields pervade the disk; gas is accelerated because the magnetic field enforces its corotation with the disk out to relatively large distances. Such centrifugally driven, hydromagnetic winds are very efficient in extracting and removing angular momentum and rotational energy from the disk, although it is not clear that winds can be formed at the large distances from the central source assumed in this scenario. Another disk-wind model assumes that Alfvén waves are generated by turbulence in the disk (Hartmann and Kenyon 1985), and that the FUor winds are driven essentially by the same mechanism suggested for $\mathrm{T}$ Tauri winds (Lago 1979, 1984, DeCampli 1981, Hartmann et al. 1982). It is indeed possible that both the magnetic wave model and the rotating magnetic field acceleration mechanism is at play simultaneously. 
Detailed analysis of high-resolution line profiles can provide information on the geometry of the wind-forming region, the mass loss-rate and the temperature of the wind. Such a study was performed by Croswell et al. (1987), who did radiative transfer calculations to match observed $\mathrm{H} \alpha$ and $\mathrm{Na}$ I profiles; each of their isothermal steady-flow wind models were specified by a mass-loss rate, a wind temperature and a wind-velocity as function of radius, and were joined to a photosphere of $\mathrm{T}_{e}=$ $5000 \mathrm{~K}$ and $\log \mathrm{g}=1$. A lower limit to the mass-loss rate of $10^{-5} M_{\odot} y r^{-1}$ was found to be required in order to match the extensive blueshifted Sodium absorption troughs. This was derived assuming a slowly accelerating velocity field; a rapidly accelerating velocity law would demand even higher mass loss rates, approaching the about $10^{-4} M_{\odot} y r^{-1}$ in mass accretion required by Hartmann and Kenyon (1985) to account for the observed outburst-luminosity. But rapid wind acceleration is only needed in a spherically-symmetric geometry, where the star is required to occult the redshifted material. If instead one assumes a disk-like geometry, a slow acceleration geometry and thus a lower mass-loss rate can still be adopted. The models which simultaneously can match the observed $\mathrm{H} \alpha$ and $\mathrm{Na}$ I absorptions all indicate cool wind temperatures of about $5000 \mathrm{~K}$; at higher temperatures $\mathrm{H} \alpha$ goes into strong emission for mass-loss rates sufficient to produce the observed Na I absorption. The work of Croswell et al. (1987) thus suggests that FUor winds are cool, very massive and arise from a disk-geometry. One consequence of the low wind temperature is that thermal pressure almost certainly is not important in driving the outflow, further supporting the magnetic field models previously discussed.

There are two observations which suggest that the wind rising from the disk has radial structure. Firstly, monitoring of the $\mathrm{H} \alpha$ and $\mathrm{Na} I$ line profiles have revealed variations of the high-velocity wings on timescales of a day (Croswell et al. 1987, Pasquini and Reipurth 1990). For wind velocities up to several hundred km/sec, this is likely to imply that this fast component of the wind originates in a region only a few stellar radii in size, presumably located around the central star . Secondly, Kenyon and Hartmann (1989) observed slightly blueshifted CO $\mathrm{v}^{\prime}-\mathrm{v}^{\prime \prime}=2-0$ absorption in FU Ori and V1057 Cyg in their Fourier Transform spectra, and concluded that mass ejection from the near-infrared emitting disk regions does not exceed $10^{-7} M_{\odot} y r^{-1}$. Compared with the mass loss rates derived for the hotter, inner, optically emitting regions (Croswell et al. 1987), it appears that the mass loss rates decrease by a factor of roughly 100 as one goes from about $1-2 \mathrm{R}_{*}$ to about $10 \mathrm{R}_{*}$.

There are theoretical arguments why the main mass loss should be concentrated towards the inner disk regions. Pringle (1989) shows that if the wind is driven ultimately by accretion energy, then the wind must come from regions of sufficient depth in the gravitational well, so that enough energy per gram becomes available. $\mathrm{He}$ suggests that the wind actually originates in the boundary layer, where the accretion disk feeds into the star, and is driven by powerful toroidal magnetic fields produced by the strong shear in the boundary layer.

Little is known with certainty about the properties of the boundary layer, but it is likely to be the hottest region of the disk. Ultraviolet spectra of several FUors 
obtained with the IUE satellite have been reported by Ewald et al. (1986) and Kenyon et al. (1989). Ultraviolet absorption features suggest spectral types for FU Ori of A5 $\mathrm{I}$ and for Z CMa of F5 I. An unexpected result is that the ultraviolet absorption features are not significantly veiled, implying that a hot boundary layer contributes less than $20 \%$ of the radiation between $2600 \AA$ and 3200 . $\AA$. An understanding of the properties of the boundary layer is likely to be one of the prime goals of FUor research in the coming years.

\section{Do FUors power the Herbig-Haro flows?}

The massive, supersonic flows of material lost from a FUor during an eruption are likely to have a profound effect on the ambient medium. It has been proposed that FUor eruptions are responsible for the Herbig-Haro flows (e.g. Dopita 1978, Reipurth 1985a, 1989a).

Herbig-Haro energy sources are, when known, mostly embedded objects, detectable only at infrared wavelengths. In those cases where the source is a visible star, it is generally highly reddened. This suggests that Herbig-Haro energy sources, as a class, belong among the youngest stars known.

The Herbig-Haro jets, the best collimated subset of the Herbig-Haro flows, provide an intriguing glimpse into the recent past of their driving sources. A number of jets show multiple bow shocks (e.g. Reipurth 1989b) testifying to intermittent mass-loss episodes in their driving sources. Viewed as a group, the energy sources also show considerable variety in terms of luminosity, type and visibility. It is here of particular interest that 3 out of the 8 FUors discussed in Section 1 are associated with Herbig-Haro objects. The close association between $\mathrm{HH} 57$ and an emerging FUor has already been discussed in Section 1 (see Fig. 4). The embedded source L1551 IRS 5 produces a jet, seen in Fig. 10 in a red CCD image from Campbell et al. (1988), which has a large proper motion away from the embedded source (Neckel and Staude 1987, Campbell et al. 1988). Z CMa possesses a bipolar Herbig-Haro jet with a total extent of $3.6 \mathrm{pc}$ and large radial velocities of up to $-600 \mathrm{~km} / \mathrm{sec}$ (Poetzel et al. 1989). It is important to note that no other class of young stars can boast a similar high percentage $(\simeq 30 \%)$ of association with Herbig-Haro objects.

If FUors initiate Herbig-Haro flows, then three questions arise. Firstly, why are the remaining FUors not associated with $\mathrm{HH}$ objects? A possible explanation is that the close environment of the star plays a role in focusing the outflow sufficiently to form shocks; note that the two deepest embedded FUors, V346 Nor and L1551 IRS 5 , are both associated with Herbig-Haro objects. Secondly, why are most HerbigHaro energy sources $\mathrm{T}$ Tauri stars? That is likely to be due to differences in the timescales of the phenomena involved. Dynamical timescales for Herbig-Haro flows are typically a few thousand years, whereas timescales for the decay of FUors are likely to be of the order of a few hundred years. At any given time, the majority of 


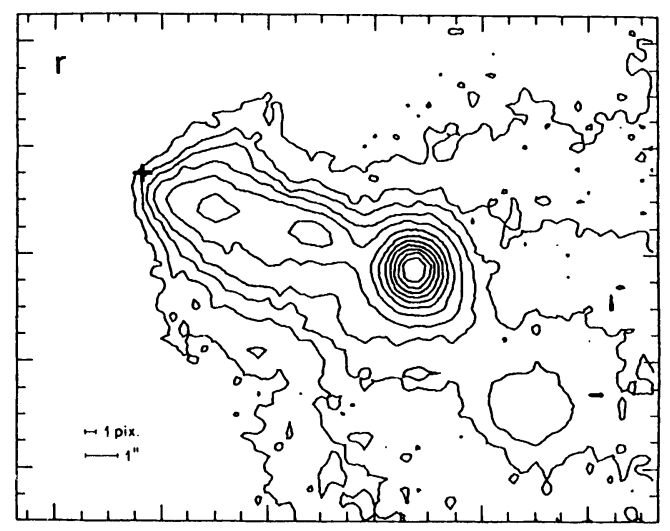

Fig. 10. The Herbig-Haro jet emanating from the embedded FUor L1551 IRS 5, whose position is indicated by a cross, as seen in a CCD image through a red broadband filter. From Campbell et al. (1988).

energy sources have therefore reverted to a stage in which the star again dominates the luminosity. Thirdly, since some Herbig-Haro jets are seen to emanate from $\mathrm{T}$ Tauri stars, which therefore still have winds sufficiently powerful and collimated to form shocks, what distinguishes these stars from the average T Tauri star? Essentially all known T Tauri-type Herbig-Haro energy sources belong to the rich emission line variety, Herbig's class 4 and 5 (Herbig 1962), suggesting that they are in a higher state of activity. Perhaps these stars are in EXor-like phases towards the end of their decay from an elevated FUor phase.

\section{FUors and pre-main sequence evolution}

Based on the number of known FUors, together with an estimate of the population of $\mathrm{T}$ Tauri stars that could give rise to FUors, and the time interval in which photographic surveys have been made, Herbig (1977) concluded that the FUor phenomenon is repetitive and occurs many times in each $\mathrm{T}$ Tauri star. Deriving a rough mean interval of 10000 years between eruptions, and assuming a T Tauri phase of $10^{6}$ years duration, Herbig suggested that a T Tauri star might experience as many as 100 eruptions. Estimates made in a different way by Hartmann and Kenyon (1985, 1987a) support Herbig's finding that FUors are repetitive.

It is probable that correct estimates of the FUor frequency cannot be derived by searching for brightenings only in visible stars. If FUors are caused by disk accretion, it seems likely that eruptions would be more frequent in the earliest phases of evolution, during which the stars are still embedded in molecular clouds and their 
disks are less depleted. Infrared sources could therefore be the most likely reservoir of future FUor eruptions. It is indeed intriguing that embedded Herbig-Haro energy sources have a very large spread in luminosity.

The early evolution of a young low-mass star may be cyclic, with occasional accretion events elevating the star into a FUor stage from which it gradually decays into a $\mathrm{T}$ Tauri phase. It has been proposed by a number of authors that even the $\mathrm{T}$ Tauri characteristics are powered by accretion. The true unperturbed underlying star may first be seen when the star reaches the weak-line $\mathrm{T}$ Tauri phase. The full evolutionary cycle would then be from FUor to T Tauri star to weak-line T Tauri star, except that in the earliest phases the star never reaches the weak-line state because of accretion, and in the late phases the massive accretion events seen as FUors become very rare or non-existent, and the star then basically cycles between the T Tauri and the weak-line $\mathrm{T}$ Tauri phase. This cyclic way of looking also at the later phases of pre-main sequence evolution can explain why some weak-line T Tauri stars still can have considerable circumstellar material.

If mass accretion rates reach $10^{-4} M_{\odot} y r^{-1}$ during a FUor event, which may last on average $10^{2}$ years, then as much as $0.01 M_{\odot}$ could be accreted in a single event. If the FUor phenomenon is repetitive, a substantial part of the stellar mass might be added from the disk during the early'evolution of the star, as proposed by Mercer-Smith et al. (1984). Even the smaller accretion-rates occurring during the $\mathrm{T}$ Tauri phases may, because of the much longer duration of these phases, have an effect on the stellar mass. Accretion may thus alter the evolution in the HR diagram significantly (Hartmann and Kenyon 1990).

FU Orionis eruptions may also have an impact on our understanding of the early solar nebula. Herbig (1977) pointed out that dust grains in the inner regions of the disk could be significantly heated, and speculated that the chondrules found in chondritic meteorites might have formed from dust melted during FUor outbursts. Models of the early solar nebula (e.g. Wood and Morfill 1988) may need to take into account the effects of cyclic heating of disk material by FUor events.

\section{Acknowledgements}

I am grateful to Luiz Paulo Vaz for hospitality at the Institute of Physics and Astronomy/UFMG in Belo Horizonte, where this review was written, and to W. Seitter and H. Duerbeck, whose efforts to secure a visum enabled me to participate in this symposium. 


\section{References}

Ambartsumian, V.A.: 1954, Comm. Byurakan Obs. No. 13

Ambartsumian, V.A.: 1971, Astrofizika 7, 557 (Astrophysics, 7, 331)

Andersson, C., Johansson, L.E.B., Winnberg, A., Goss, W.M.: 1979, Astron. Astrophys. 80, 260

Basri, G., Bertout, C.: 1989, Astrophys. J. 341, 340

Bastian, U., Mundt, R.: 1985, Astron. Astrophys. 144, 57

Bertout, C., Krautter, J., Möllenhoff, C., Wolf, B.: 1977, Astron. Astrophys. 61, 737

Bertout, C., Basri, G., Bouvier, J.: 1988, Astrophys. J. 330, 350

Cabrit, S., Edwards, S., Strom, S.E., Strom, K.M.: 1990, in press

Campbell, B., Persson, S.E., Strom, S.E., Grasdalen, G.L.: 1988, Astron. J. 95, 1173

Carr, J.S., Harvey, P.M., Lester, D.F.: 1987, Astrophys. J. 321, L71

Chavarria-K., C.: 1977, Astron. Astrophys. 79, L18

Chalonge, D., Divan, L., Mirzoyan, L.V.: 1982, Astrofizika, 18, 263 (Astrophysics 18, 263)

Clarke, C.J., Lin, D.N.C., Papaloizou, J.C.B.: 1989, Monthly Notices Roy. Astron. Soc. 236, 495

Cohen, M.: 1973, Monthly Notices Roy. Astron. Soc. 161, 105

Cohen, M.: 1975, Monthly Notices Roy. Astron. Soc. 173, 279

Cohen, M.: 1980, Astron. J. 85, 29

Cohen, M., Harvey, P.M., Schwartz, R.D.: 1985, Astrophys. J. 296, 633

Cohen, M., Bieging, J.H.: 1986, Astron. J. 92, 1396

Cohen, M., Dopita, M.A., Schwartz, R.D.: 1986, Astrophys. J. 302, L55

Cohen, M., Schwartz, R.D.: 1987, Astrophys. J. 316, 311

Cohen, M., Emerson, J.P., Beichman, C.A.: 1989, Astrophys. J. 339, 455

Covino, E., Terranegra, L., Vittone, A.A., Russo, G.: 1984, Astron. J. 89, 1868

Croswell, K., Hartmann, L., Avrett, E.H.: 1987, Astrophys. J. 312, 227

Cudworth, K.M., Herbig, G.H.: 1979, Astron. J. 84, 548

DeCampli, W.M.: 1981, Astrophys. J., 244, 124

Dopita, M.A.: 1978, Astron. Astrophys. 63, 237

Duncan, D.K., Harlan, E.A., Herbig, G.H.: 1981, Astron. J. 86, 1520

Elias, J.H.: 1978, Astrophys. J. 223, 859

Ewald, R., Imhoff, C.L., Giampapa, M.S.: 1986, In New Insights in Astrophysics, ESA SP-263, p. 205

Finkenzeller, U., Jankovics, I.: 1984, Astron. Astrophys. Suppl. 57, 285

Finkenzeller, U., Mundt, R.: 1984, Astron. Astrophys. Suppl. 55, 109

Gahm, G.F., Welin, G.: 1972, IBVS No. 741

Gasparian, K.G., Ohanian, G.B.: 1989, Inf. Bull. Var. Stars 3327 
Goodrich, R.W.: 1987, Publ. Astron. Soc. Pacific 99, 116

Götz, W.: 1980, Mitt. Ver. St. 8, 143

Gottlieb, E.W., Liller, Wm.: 1978, Astrophys. J. 225, 488

Graham, J.A.: 1983, IAU Circ. $\mathbf{3 7 8 5}$

Graham, J.A., Frogel, J.A.: 1985, Astrophys. J. 289, 331

Grasdalen, G.: 1973, Astrophys. J. 182, 781

Haro, G.: 1971, IBVS No. 565

Hartmann, L., Edwards, S., Avrett, E.H.: 1982, Astrophys. J. 261, 279

Hartmann, L., Kenyon, S.J.: 1985, Astrophys. J. 299, 462

Hartmann, L., Kenyon, S.J.: 1987a, Astrophys. J. 312, 243

Hartmann, L., Kenyon, S.J.: 1987b, Astrophys. J. 322, 393

Hartmann, L., Kenyon, S.J.: 1990, Astrophys. J. 349, 190

Hartmann, L., Kenyon, S.J., Hewett, R., Edwards, S., Strom, K.M., Strom, S.E., Stauffer, J.R.: 1989, Astrophys. J. 338, 1001

Herbig, G.H.: 1958, Astrophys. J. 128, 259

Herbig, G.H.: 1960, Astrophys. J. Suppl. 4, 337

Herbig, G.H.: 1962, Advances in Astron. Astrophys. 1, 47

Herbig, G.H.: 1966, Vistas in Astronomy 8, 109

Herbig, G.H.: 1977, Astrophys. J. 217, 693

Herbig, G.H.: 1989, in ESO Workshop on Low Mass Star Formation and Pre-Main Sequence Objects, ed. B. Reipurth, p. 233

Ibragimov, M.A., Shevchenko, V.S.: 1987, Astrophysics 27, 337

Kenyon, S.J., Hartmann, L., Hewett, R.: 1988, Astrophys. J. 325, 231

Kenyon, S.J., Hartmann, L.: 1989, preprint

Kenyon, S.J., Hartmann, L.: 1990, Astrophys. J. 349, 197

Kenyon, S.J., Hartmann, L., Imhoff, C.L., Cassatella, A.: 1989, Astrophys. J. 344,

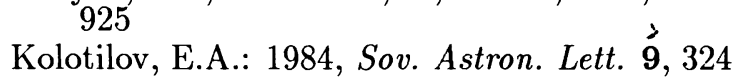

Kolotilov, E.A.: 1987, Sov. Astron. Lett. 13, 16

Kolotilov, E.A., Petrov, P.P.: 1983, Sov. Astron. Lett. 9, 92

Kolotilov, E.A., Petrov, P.P.: 1985, Sov. Astron. Lett. 11, 358

Kopatskaya, E.N.: 1984, Astrophysics 20, 138

Koresko, C.D., Beckwith, S.V.W., Sargent, A.I.: 1989, Astron. J. 98, 1394

Lada, C.J., Black, J.H.: 1976, Astrophys. J. 203, L75

Larson, R.B.: 1983, Rev. Mexicana Astron. Astrof. 7, 219

Lago, M.T.V.T.: 1979, Ph.D. thesis, Univ. of Sussex, Brighton

Lago, M.T.V.T.: 1984, Monthly Notices Roy. Astron. Soc. 210, 323

Leinert, Ch., Haas, M.: 1987, Astron. Astrophys. 182, L47

Levreault, R.M.: 1983, Astrophys. J., 265, 855 
Lin, D.N.C., Papaloizou, J.: 1985, in Protostars and Planets II, eds. D.C. Black and M.S. Matthews, Univ. of Arizona Press, Tucson, p. 981

Lo, K.Y., Bechis, K.D.: 1973, Astrophys. J. 185, L71

Lo, K,Y., Bechis, K.D.: 1974, Astrophys. J. 190, L125

Lynden-Bell, D., Pringle, J.E.: 1974, Monthly Notices Roy. Astron. Soc. 168, 603

Mazzitelli, I.: 1989, in ESO Workshop on Low Mass Star Formation and Pre-Main Sequence Objects, ed. B. Reipurth, p. 433

Mendoza, E.E.: 1971, Astrophys. J., 169, L117

Mercer-Smith, J.A., Cameron, A.G.W., Epstein, R.I.: 1984, Astrophys. J. 279, 363

Mirzoyan, L.V., Melikian, N.D., Natsvlishvili, R.Sh.: 1988, Astrofizika 28, 540 (Astrophysics 28, 320)

Moneti, A., Forrest, W.J., Pipher, J.L., Woodward, C.E.: 1988, Astrophys. J. 327, 870

Mould, J.R., Hall, D.N.B., Ridgway, S.T., Hintzen, P., Aaronson, M.: 1978, Astrophys. J. 222, L123

Mundt, R., Stocke, J., Strom, S.E., Strom, K.M., Andersson, E.R.: 1985, Astrophys. J. 297, L41

Neckel, J., Staude, H.J.: 1987, Astrophys. J. 322, L27

Pasquini, L., Reipurth, B.: 1990, in preparation

Paczynski, B.: 1976, Qly. J. Roy. Astr. Soc. 17, 25

Parsamian, E.S., Gasparian, K.G.: 1987, Astrofizika 27, 447 (Astrophysics 27, 598)

Pringle, J.E.: 1989, Monthly Notices Roy. Astron. Soc. 236, 107

Poetzel, R., Mundt, R., Ray, T.P.: 1989, Astron. Astrophys. 224, L13

Pudritz, R.E., Norman, C.A.: 1983, Astrophys. J. 274, 677

Pudritz, R.E., Norman, C.A.: 1986, Astrophys. J. 301, 571

Reipurth, B.: 1985a, Astron. Astrophys. 143, 435

Reipurth, B.: 1985b, in ESO-IRAM-Onsala Workshop on (Sub)-millimeter Astronomy, ed. P.A. Shaver and K. Kjär, p. 459

Reipurth, B.: 1989a, in ESO workshop on Low Mass Star Formation and Pre-Main Sequence Objects, ed. B. Reipurth, p. 249

Reipurth, B.: 1989b, Nature 340, 42

Reipurth, B.: 1990, in preparation

Reipurth, B., Olberg, M., Booth, R.: 1990, in preparation

Sargent, A.I., Beckwith, S., Keene, J., Masson, C.: 1988, Astrophys. J., 333, 936

Schwartz, R.D., Jones, B.F., Sirk, M.: 1984, Astron. J. 89, 1735

Schwartz, R.D., Snow, T.P.: 1972, Astrophys. J., 177, L85

Shakura, N.I., Sunyaev, R.A.: 1973, Astron. Astrophys. 24, 337

Shanin, G.I.: 1979, Sov. Astron. 23, 158

Simon, T., Joyce, R.R.: 1988, Publ. Astron. Soc. Pacific 100, 1549 
Smith, H.A., Thronson, H.A., Lada, C.J., Harper, D.A., Loewenstein, R.F., Smith, J.: 1982, Astrophys. J. 258, 170

Snell, R.L., Loren, R.B., Plambeck, R.L.: 1980, Astrophys. J. 239, L17

Stocke, J.T., Hartigan, P.M., Strom, S.E., Strom, K.M., Andersson, E.R., Hartmann, L.W., Kenyon, S.J.: 1988, Astrophys. J. Suppl. 68, 229

Strom, K.M., Strom, S.E., Kenyon, S.J., Hartmann, L.: 1988, Astron. J. 95, 534

Strom, S.E., Strom, K.M., Vrba, F.J.: 1976, Astron. J. 81, 320

Wachmann, A.A.: 1939, Beob. Zirk. 21, 12

Wachmann, A.A.: 1954, Zeitschrift f. Astrophysik 35, 74

Welin, G.: 1971, Astron. Astrophys. 12, 312

Welty, A.D., Strom, S.E., Strom, K.M., Hartmann, L., Kenyon, S.J., Grasdalen, G.L., Stauffer, J.R.: 1990, Astrophys. J. 349, 328

Winnberg, A., Graham, D., Walmsley, C.M., Booth, R.S.: 1981, Astron. Astrophys. 93, 79

Wood, J.A., Morfill, G.E.: 1988, in Meteorites and the Early Solar System, eds. J.F. Kerridge, M.S. Matthews, University of Arizona Press, p. 329

GAHM: Just a comment on your note on the unusual strength of Ba II at $6496 \mathrm{~A}$. This feature is very strong in red supergiants, so may be this is in line with your picture of line formation in a low pressure disk. 